\title{
Land Covers Classification for Remote Sensing Images Based on Spectral and Textural Information
}

\author{
Wei ZHAO, Shumei CUI \\ Harbin Institute of Technology, Harbin, China.
}

\begin{abstract}
In this paper, the classifiers for land/water and natural land/artificial land combined the texture measures with spectral analysis for remote sensing images have been built. The specific recognition has been designed in order to take advantages of both analyses. Moreover, coastal line development analysis, the length or ratio of natural/artificial seacoast, could be calculated automatically. The use of red/infrared spectral analysis refines the boundary of classes; meanwhile the merging of co-occurrence matrix texture analysis and spectral information has improved the accuracy of the three-class labeling.
\end{abstract}

Keywords: Remotely Sensed Images; Texture Analysis; Grey level Cooccurrence Matrix; Spectral Feature

\section{Introduction}

Machine interpretation using pattern recognition technique for Remote Sensing (RS) images could not only liberate plenty of human resources, but improve results on the efficiency in certain aspects.

Classifying various land cover, such as soil, trees, grass, naked rock, river, or sea in remote sensing images, is a fairly significant research in remote sensing imagery understanding. The remotely sensed images, which characterize the differential of land cover types, are ob- tained from the transform of the response reflecting varies band of electromagnetic radiation. Evidences distinguishing these coverings on land could be the differentia of pixel grey level and that of the homogeneity as well in the images. By computer-aided labeling each pixel or homogeneity region with corresponding properties, interested images or parts of the image in study could be interpreted specifically and effectively.

For remotely sensed images purposes, texture is a measure of the spatial variation of digital image spectral brightness or digital number (DN) ${ }^{[1]}$. It is a great impelling and fictile feature and tool that most of studies experiment use it in variation ways. Haralick gives the following definition of texture in $1979^{[2]}$, "Image texture is described by the number and types of its primitives and the spatial organization or layout of its primitives. The spatial organisation may be random, may have a pairwise dependence of one primitive on a neighbouring primitive, or may have a dependence of a primitive at a time. The dependence may be structural, probabilistic or functional”.

It is suggested that the most practical approach is to analyze each data type separately, by techniques optimized to that data's characteristics, and then to fuse at the label level ${ }^{[1]}$. Synergy between spatial variability (e.g., texture) and spectral brightness (e.g., tone) has great potential to overcome the problem

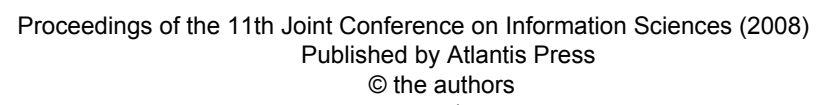


of misclassification of land cover in fine spatial resolution imagery ${ }^{[3]}$.

In this paper, the land-type and watertype patterns in remote sensing images are interested and distinguished by texture feature, the grey level co-occurrence matrix (GLCM), and spectral information, the red-light and infrared band, analyzing respectively. Followed by the combination of the two results, type-labeling and conclusion are made.

\section{Land/Water Spectral Data Analysis}

Deep water or clear water generally takes on the color of blue or glaucous. The blue or green band reflectivity of the water is about $4 \% \sim 5 \%$. Compared to it, that of the red light drops to $2 \% \sim 3 \%$, while the incidence energy of near infra red (IR) and shortwave IR are almost absorbed entirely. On the other hand, the red and IR spectral on vegetation, soil and naked rock are reflected in an obviously degree.

Moreover, there are textures on land covering, caused by urban establishment or natural objects, such as roads, buildings, rivers or woods, which could be extracted from various types of cities or vegetations. But the similar features are hardly gotten from water type coverings, especially from equable seas, lakes or rivers. Therefore, merging spectral features and textural information in a way could effectively and exactly label the land and water regions in remote sensing images.

\section{Grey Level Co-occurrence Matrix}

Pixel pairs with equable or unequal grey level, which are apart at a distance in a 3-dimension gray-level space, must abide by a certain statistical rule of united distribution. If the regularity of any pair of the pixels has been found in an image, it is out of question good for image measure and textural analysis.

Grey level co-occurrence matrix (GLCM) proposed by Haralick et al. in 1973 turned out to be one of the important textural measure tool. It has been applied in several remote sensing applications ${ }^{[4]}$. Based on the second order conditional probability density function, GLCM reflects the integrated information of direction, distance, range or speed of varying in an image by calculating the relativity of any pair of points in a direction at a distance.

Angular second moment, contrast, correlation, sum variance, sum average, entropy, sum entropy, difference entropy, dissimilarity etc are the textural features could be obtained from GLCM, which describe relation between different pixels and does not over-emphasize field boundaries. Meanwhile, these features are sensitive to directionality but not to noise [4]. Whereas, they are computationally intensive and of similarity among the statistics derived from co-occurrence matrix

The matrix records the numbers of pixels with a grey scale $i$ which are separated from pixels with a grey scale $j$ by a particular distance and direction. Assume $x, y$ axes are the coordinates of image $I, Z$ indicates the grey level of pixels, then we have:

$$
(k, l),(m, n) \text {-- Coordinates of pixels to }
$$
be measured.

$$
N_{x}, N_{y}-- \text { Number of pixels in }
$$

$x, y$ direction;

$$
\begin{aligned}
& L_{x}=\left\{1,2, \mathrm{~L}, N_{x}\right\}-- \text { Horizontal domain; } \\
& L_{y}=\left\{1,2, \mathrm{~L}, N_{y}\right\}-- \text { Vertical domain; } \\
& N_{g}-\text { - Quantified grey level; } \\
& G=\left\{1,2, \mathrm{~L}, N_{g}\right\}-\text { Set of quantified }
\end{aligned}
$$

grey level; 
$L_{y} * L_{x}$--pixel set of image $I$. The grey

level of each pixel is from the set of $G$ see formula (1):

$$
I: L_{y} * L_{x} \rightarrow G
$$

Formula (2) is the GLCM in four directions of $0^{\circ}, 45^{\circ}, 90^{\circ}, 135^{\circ}$. The distance of any pair of pixels is $d$ :

$$
\begin{gathered}
P\left(i, j, d, 0^{\circ}\right)=\#\{[(k, l),(m, n)] \mid \\
k-m=0,|l-n|=d, I(k, l)=i, I(m, n)=j\} \\
P\left(i, j, d, 45^{\circ}\right)=\#\{[(k, l),(m, n)] \mid \\
k-m=d, l-n=d, I(k, l)=i, I(m, n)=j\} \\
P\left(i, j, d, 90^{\circ}\right)=\#\{[(k, l),(m, n)] \mid \\
\quad k-m=d, l-n=0, I(k, l)=i, I(m, n)=j\} \\
P\left(i, j, d, 135^{\circ}\right)=\#\{[(k, l),(m, n)] \mid \\
\quad k-m=-d, l-n=-d, I(k, l)=i, I(m, n)=j\}
\end{gathered}
$$

where \# is the number of elements in set $G$ and $P$ is the matrix of GLCM of corresponding direction. It is obviously that these matrixes are symmetrical ones, namely $P(i, j, d, \theta)=P(j, i, d, \theta)^{[5]}$.

In this paper, the following processes are taken to extract the remotely sensed image textural information:

1. Grey-level compression from 256 to 8 or 16 to lessen the amount of computation.

2. Setting the size of non-overlapping window from $7 \times 7,9 \times 9,11 \times 11$, $13 \times 13$, etc;

3. Calculating the four directions GLCM in $0^{\circ}, 45^{\circ}, 90^{\circ}, 135^{\circ}$ respectively;

4. Standardizing the GLCM of an image to be analyzed. $\hat{p}(i, j)$ is instead of $p(i, j)$ in the matrix, where $\hat{p}(i, j)=p(i, j) / R$, and $R$ is standardizing constant related to directions. Since there are $2\left(N_{x}-1\right)$ adjacent pixel pairs in $0^{\circ}$ a row and $2 N_{y}\left(N_{x}-1\right)$ pairs of the whole im- age, $R=2 N_{y}\left(N_{x}-1\right)$. The $R$ in $90^{\circ}$ is $2 N_{x}\left(N_{y}-1\right)$, in $45^{\circ}, 135^{\circ}$ is $2\left(N_{y}-1\right)\left(N_{x}-1\right)$ in a similar way.

5. Traversal the whole image to get the textural features in the window, and make it as the centre pixel's feature.

In this paper, the grey-level was compressed to 8; to balance the computational intensive and the performance of class labeling, the size of window is fixed to $11 \times 11$.

Meanwhile, the said features extracted from GLCM, in which the redundancy and repetitious data exist unavoidably, could express certain textural information. Not all but several of them, such as angular second moment, contrast, correlation and the inverse difference moment features based on GLCM, are used as perfectly representative and independence textural parameters ${ }^{[6]}$. They also have good performance in this paper.

\section{Spectrum Feature Extraction and Training}

When reflect red/infra-red band, water gives pretty low reflection rate, while land covers with abundant light supply (non-shadow region) showed generally high rate.

Distill the red/infra-red measure from each of the land/water training samples and get average and extremums of each image. The bold-face numbers in TABLE I are respectively the average of minimum average of land samples, 27.2774; that of maximum average of land samples, 178.7181; that of minimum average of water samples, 7.4523; that of maximum average of water samples, 27.3615.

The average of minimum average of land samples is nearly equal to the average of maximum average of water samples. In the subset of training samples, which is showed in TABLE I and Fig.1

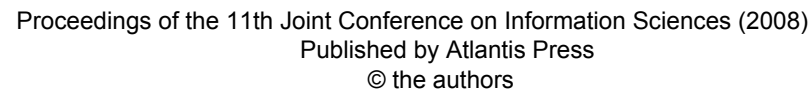


and Fig.2, the value is about 27. It could be test and set as threshold in the task of land/water region recognition.

Under the whole training set, in which 39/52 water/land-region samples are included, the threshold gather statistics as 32. With it, almost all the water could be recognized with red-light spectral information.

Two classifiers, in which GLCM and spectral features are used respectively, have been built based on the least error rate Bayesian discriminant function with multi-normal distribution:

$g_{i}(x)=-\frac{1}{2}\left(x-\mu_{i}\right)^{T} \Sigma_{i}^{-1}\left(x-\mu_{i}\right)-\frac{d}{2} \ln 2 \pi-\frac{1}{2} \ln \left|\Sigma_{i}\right|+\ln P\left(\omega_{i}\right)$

where, $i$ is the tag of class, $i=0,1 ; T$ is the transposition of matrix, $\sum_{i}$ is covariance matrix, $p\left(\omega_{i}\right)$ is prior probability of class $i, \mu_{i}$ is the average vector.

Ignoring the items unrelated with class $i$, the function is as follow: where $\sigma$ is the average

Table 1. Parameter comparison of Redspectral Feature in part of training samples.

\begin{tabular}{|c|c|c|c|c|c|c|}
\hline \multicolumn{2}{|c|}{$\begin{array}{l}\text { Training } \\
\text { Samples }\end{array}$} & Min & $\begin{array}{l}\text { Line-mini } \\
\text { Ave }\end{array}$ & Ave & Max & $\begin{array}{c}\text { Line-max } \\
\text { Ave }\end{array}$ \\
\hline \multirow{15}{*}{ land } & 1 & 0 & 32.2661 & 129.2517 & 234 & 199.2477 \\
\hline & 2 & 15 & 28.8440 & 85.2683 & 244 & 190.3211 \\
\hline & 3 & 0 & 16.1284 & 113.3348 & 206 & 187.3486 \\
\hline & 4 & 0 & 13.5229 & 71.1981 & 217 & 174.7248 \\
\hline & 5 & 0 & 10.1284 & 68.7048 & 228 & 186.3578 \\
\hline & 6 & 0 & 11.2936 & 50.7630 & 157 & 118.2294 \\
\hline & 7 & 0 & 19.3303 & 107.2764 & 255 & 231.7798 \\
\hline & 8 & 26 & 58.8532 & 127.0319 & 245 & 187.8440 \\
\hline & 9 & 12 & 28.2294 & 117.8535 & 254 & 199.1468 \\
\hline & 10 & 8 & 30.1743 & 90.7336 & 241 & 188.6789 \\
\hline & 11 & 24 & 40.2663 & 59.2722 & 87 & 74.7160 \\
\hline & 12 & 0 & 11.2920 & 76.2255 & 226 & 173.5839 \\
\hline & 13 & 31 & 51.4949 & 103.6763 & 255 & 203.7710 \\
\hline & 14 & 15 & 30.0597 & 89.4260 & 255 & 186.3035 \\
\hline & - & - & 27.2774 & - & - & 178.7181 \\
\hline \multirow{11}{*}{ water } & 1 & 4 & 7.4954 & 13.0328 & 41 & 24.4220 \\
\hline & 2 & 13 & 18.3303 & 25.0185 & 99 & 35.0092 \\
\hline & 3 & 8 & 12.7156 & 19.1833 & 26 & 23.6972 \\
\hline & 4 & 0 & 4.6055 & \begin{tabular}{|l|l|}
10.2499 \\
\end{tabular} & 25 & 16.6606 \\
\hline & 5 & 0 & 3.4404 & 12.9600 & 40 & 23.0183 \\
\hline & 6 & 0 & 3.7706 & 12.6356 & 25 & 21.3486 \\
\hline & 7 & 10 & 13.2110 & 30.2781 & 113 & 78.6606 \\
\hline & 8 & 0 & 0 & 3.5237 & 15 & 10.2202 \\
\hline & 9 & 0 & 0 & 4.2550 & 28 & 12.6330 \\
\hline & 10 & 9 & 10.9541 & \begin{tabular}{|l|}
19.7344 \\
\end{tabular} & 30 & 27.9450 \\
\hline & - & - & 7.4523 & - & - & 27.3615 \\
\hline
\end{tabular}

of inner-class feature standard deviation.

It is usually assumed in remotely sensing application that the possibilities observed classes is equal ${ }^{[7]}$. Thus the classifier could be further simplified as:

$$
g_{i}(x)=-\frac{1}{2 \sigma^{2}} \sum_{j=1}^{d}\left(x_{j}-\mu_{i j}\right)^{2}-d \ln \sigma+\ln P\left(\omega_{i}\right)
$$

where $\sigma$ is the average of inner-class feature standard deviation.

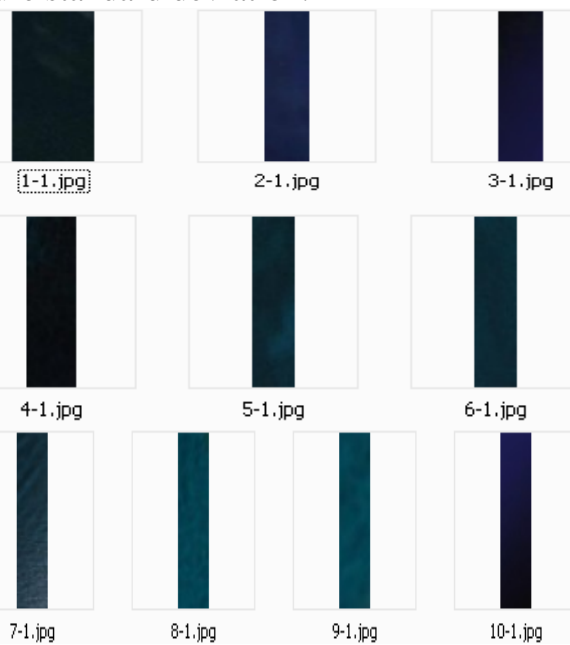

Fig. 1 Part of the training samples of water.

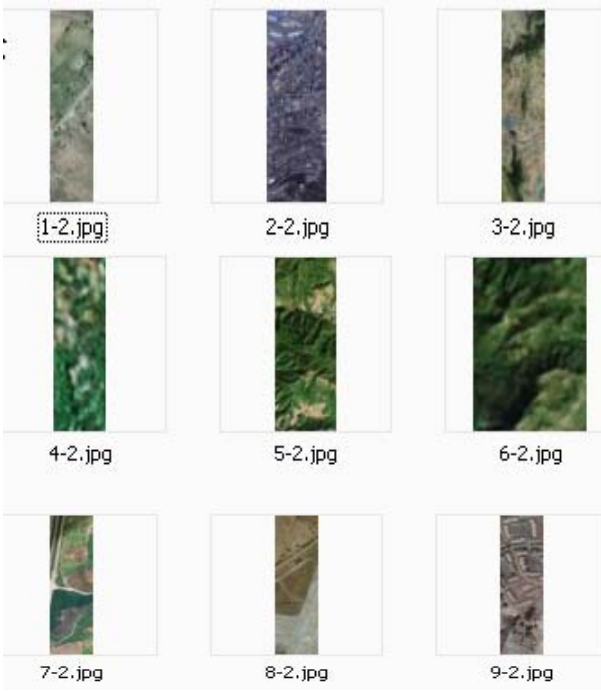

Fig. 2 Part of the training samples of water/land class.

It is usually assumed in remotely sensing application that the possibilities ob- 
served classes is equal ${ }^{[7]}$. Thus the classifier could be further simplified as:

$g_{i}(x)=-\frac{1}{2 \sigma^{2}} \sum_{j=1}^{d}\left(x_{j}-\mu_{i j}\right)^{2}-d \ln \sigma$

The training stage has been carrying out separately. Testing sample is $100 \mathrm{im}$ ages in which water/land patterns coexisted. Fig. 3 and Fig. 4 give part of demonstrations of testing result.

Shadow or rather smooth regions in land image are generally labeled as water because of the sparseness of textural measure, while the land regions under clear or shallow water are prone to be recognized as land. Fig.3 c is the result that classifier with only texture feature would make.

It is obviously to find that the island area including the underwater part had been labeled as land in Fig. 3 c). Moreover, the lake on the land in Fig. 4 has been labeled as land and that spectral classifier keep it faithfully.

The d) in the figures is the merging result of the b) and c). From the final result, the refined boundary owing to spectral classifier is fairly good, and small regions of water or land are also retained correctly.
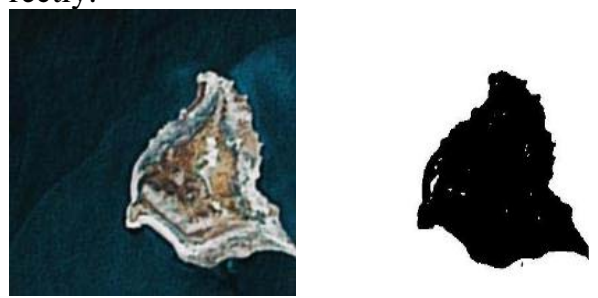

a) Original Image

b) Recognized by spectrum feature

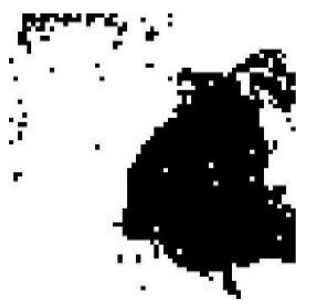

c) Recognized by texture feature

Fig.3 Demo-1of recognition results

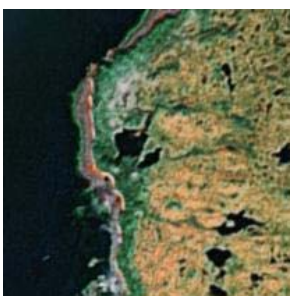

a) Original Image

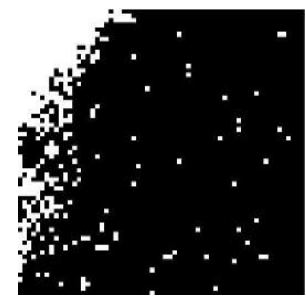

c) Recognized by texture feature

Fig.4 Demo-2 of recognition results

\section{Coastal Land Cover Distinguish And Analysis}

Monitoring landscape changes near coastal area allow human find out the environmental transformations at local, regional, even global scales.

The following tablets are the parts of the two classes' samples cut from coastal RS images. See Fig. 5 a) and b). A simple state of coastal land cover identification is narrated below.

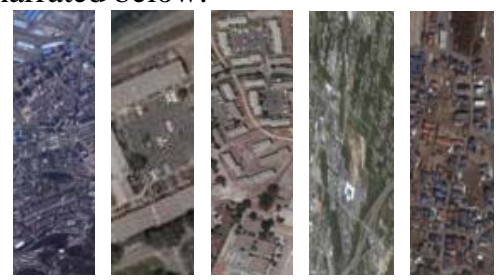

a) Samples of coastal urban area

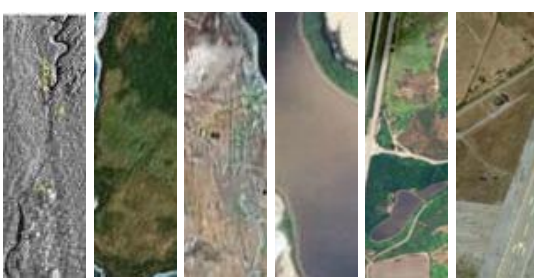

b) Samples of natural coastal land cover Fig.5 Coastal land cover samples 


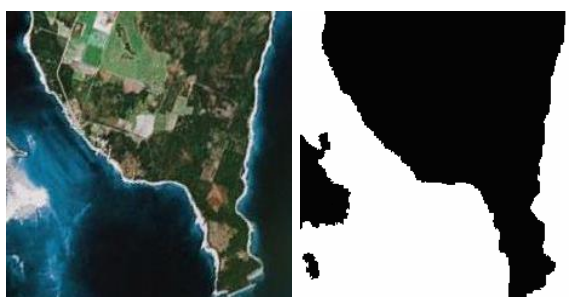

Fig. 6 Demo of Coastal area Original Image and Final result

After the Land/Water analysis, Fig. 6, the boundary could be clear and the length of coastal line could obtain easily, e.g. by counting the pixel number of the boundary. To distinguish the type of land cover near the water, a zonal boundary was made extending on the black area (the land area), see the grey point in Fig.7. The width of the zone is about one eighth or one tenth of the images size. It depends on the interesting area scope.

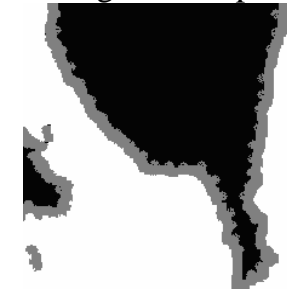

Fig. 7 Illustration of zonal boundary

The zonal boundary, as the target, was input into the second classifier which is the same as the land/water classifier but trained by the coastal land cover samples in Fig. 5, with the threshold of 16.7 getting from the 54 and 38 samples of urban and natural land. After determination and labeling, the ratio of natural/urban zonal area has been calculated.

However, for the area that is amphibolous, no matter what scale researchers take for consideration, the spatial scales or temporal ones, natural-area and urban area are usually confusion with each other in that textural or homogeneity information is unconvincing in land class labeling. Machinery interpretation of RS images requires an evolution of remote sensing and Geographic Information Sys- tems technology which has allowed for many types of data integration.

\section{References}

[1] Richards J.A. “Analysis of Remotely Sensed Data: The Formative Decades and the Future”, IEEE Transactions on Geosciences and Remote Sensing, 2005, 43(3), pp.422-432.

[2] Haralick, R.M. "Statistical and structural approaches to texture”, Proceedings of the IEEE, 1979, 67 (5):pp. 786-804.

[3] Stein, A., Bastiaanssen, W.G.M., De Bruin, S., Cracknell, A.P., Curran, P.J., Fabbri, A.G., Gorte, B.G.H., Van, Groenigen, J.W., Van Der Meer, F.D. \& Saldana, “A. Integrating spatial statistics and remote sensing, International Journal of Remote Sensing” 1998,19: pp. 1793-1814.

[4] Steven M. De Jong, Freek D. Van Der Meer, Remote Sensing Image Analysis: Including the Spatial Domain, Kluwer Academic Publishers, 2004.

[5] SONG Yang, WAN You-chuan, CHEN Peng, "Textural Features Analysis Based on GLCM in TM Thermal Infrared Remotely Sensed Images”, Remote Sensing Information, 2006, vol.4, pp. 24-26.

[6] SHENG Hui , YIN Shoujing "The Application of RS and GIS in the Research of Wetland”, Remote Sensing Information, 2006, vol.2, pp.46-49

[7] L.L.Cui, Integrative Analysis and Evaluation of the Interpretation Features in Remote Sensing Image. Chinese Academy of Science Doctoral Thesis. 2005, pp.28-30. 